\section{Mohr Circle}

Jeffrey R. Keaton

Amec Foster Wheeler, Los Angeles, CA, USA

\section{Definition}

A graphical representation of normal stresses and shear stresses was developed by Professor Otto Mohr (1835-1918) who recognized that the equations could be rearranged into the form describing a circle:

$$
\begin{gathered}
{\left[\sigma_{\beta}-\left(\frac{\sigma_{x}+\sigma_{y}}{2}\right)\right]^{2}+\left[\tau_{\beta}-0\right]^{2}} \\
=\left[\sqrt{\left(\frac{\sigma_{x}-\sigma_{y}}{2}\right)^{2}+\tau_{x}^{2}}\right]^{2}
\end{gathered}
$$

where the second part of the right-hand term is the $x$ coordinate of the circle center, the second part of the middle term is the $y$ coordinate of the circle center, and the term inside the right-hand bracket is the circle radius. The Mohr circle graph contains three circles in a plane stress example: $\sigma_{2}=0$. A similar graph showing three-dimensional information would have two additional circles inset into each of the three circles shown that intersect the normal stress $x$ axis at the value of $\sigma_{2}$ with diameters equal to $\left(\sigma_{1}-\sigma_{3}\right)$ (shown), $\left(\sigma_{1}-\sigma_{2}\right)$, and $\left(\sigma_{2}-\sigma_{3}\right)$. The smaller circles that intersect the origin are uniaxial compression and uniaxial tension. The dotdash lines that are tangent to the circles denote the Mohr-Coulomb failure envelope; $\phi$ is the angle of internal friction. The inset graph displays positions of Plane $x$, Plane $y$, and Plane $\beta$ in the largest Mohr stress circle. A similar construction can be made to show the Mohr strain circle.

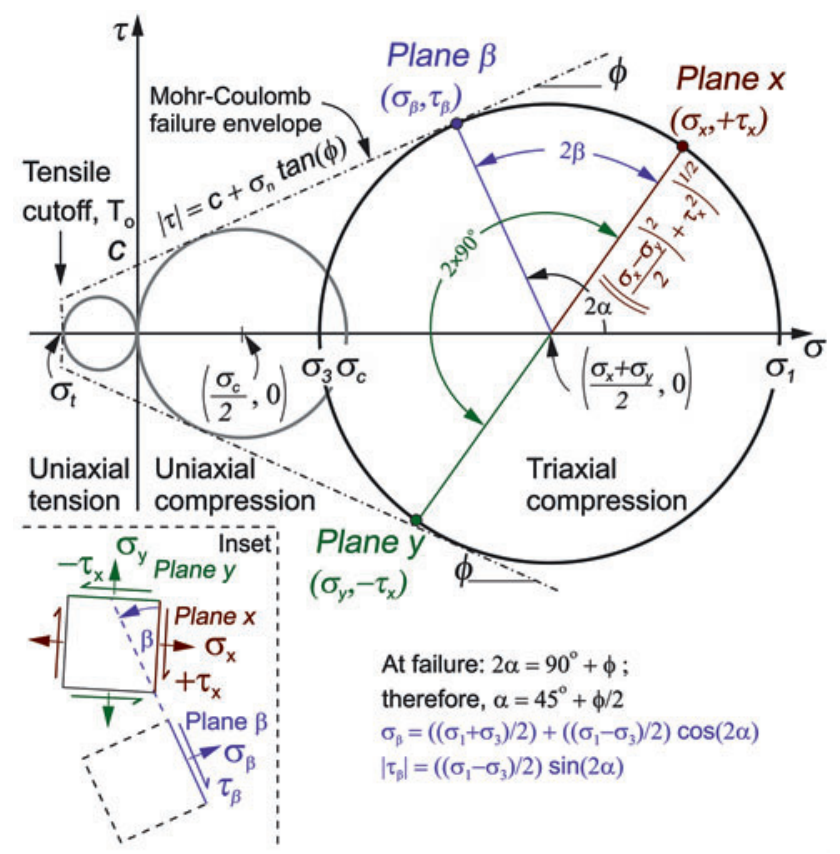

